\title{
Wireless Walking Stick with Heart Attack Detection
}

\author{
Harsha C. Puranik ${ }^{1}$, S. S. Kataria ${ }^{2}$ \\ PG Student, Electronics Department, Amrutvahini College of Engineering, Sangamner, India ${ }^{1}$ \\ Assistant Professor, Electronics Department, Amrutvahini College of Engineering, Sangamner, India ${ }^{2}$
}

\begin{abstract}
After a heart attack, the first few hours are critical in saving much of the dying heart muscle which are starving due to lack of oxygen supply and preventing permanent heart damage. The symptoms vary from individual to individual and the most common reason for critical delays is medical treatment is patient unawareness and lack of early warning. It is possible to detect the onset of a heart attack and inform the doctor or person concerned. By implanting a chip into the body, the patient need not worry about device operation. The patient will only be required to carry a cell phone equipped with Bluetooth and GPS. As soon as the implanted chip detects a heart attack, it will alert the cell phone which in turn will automatically call for help and provide the patient's location. Thus it helps in providing early heart attack detection so that the patient can get medical attention within the first few critical hours. It consists of three basic sub projects ECG Circuit, Analysis Algorithm, GPS module, GSM module and microcontroller. The first unit is worn on the user's wrist captures abnormal heart beat signal from the patient, and the rest units are installed in the stick. The microcontroller on the stick runs a heart attack algorithm. Warning is given out to the person about his heart condition. The emergency calling system calls for medical help at the moment of heart attack. The effectiveness of the proposed method is confirmed by experiments on a commercially available walking stick. Each of the subprojects responded positively. The proposed Walking Stick with Heart Attack Detection is cost effective and can save the lives of millions of old people by helping them in getting the earliest medical help in the condition of heart attack. Here we use GPS device and GSM module so at a worst conditions it will directly call 108 emergency and patient's life will be saved.
\end{abstract}

Keywords: ECG Circuitry, Microcontroller, GPS Module, GSM Module.

\section{INTRODUCTION}

The National Heart, Lung, and Blood Institute [2] states that "more than a million persons in the U.S. have a heart attack and about half $(515,000)$ of them die in each year. About one-half of those who die do so within 1 hour of the start of symptoms and before reaching the hospital". A heart attack happens to a person when the blood flow and oxygen supply to heart muscle is blocked, and it is mostly caused by the Coronary Artery Disease (CAD) [2].CAD occurs when the arteries that supply blood to the heart Causes of Heart Attack:

muscle (coronary arteries) become hardened and narrowed A coronary attack (heart attack) occurs when the blood [3]. It often causes irregular heart beat or rhythm by flow to a part of the heart is blocked (often by a blood blocking blood stream [2]. The National Heart, Lung, and clot). This happens because coronary arteries that supply Blood Institute [2] suggest that "everyone should know the the heart with blood slowly become thicker and harder warning signs of a heart attack and how to get emergency from a build up of fat, cholesterol and other substances, help".

called plaque.

The symptoms of heart attack can be detected by observing electrocardiogram (ECG) waveform. An ECG is an electrical recording of the heart and is used in the investigation of heart disease [4]. An electrical impulse initiates muscle contraction, which results in heart beating. The spacing between pulses provides a measure of the heart's rhythm, whereas the height of the pulses is an indicator of pumping strength [1]. By observing the ECG waveform, the heart condition of the patients can be explained by doctors.

The ECG Library shows many samples of abnormal ECG waveform, and they are mostly collected from aged people

If the plaque breaks open and a blood clot forms that blocks the blood flow, a heart attack occurs. Then the heart muscle supplied by that artery begins to die. Damage increases the longer an artery stays blocked. Once that muscle dies, the result is permanent heart damage.

Warning Signs of Heart Attack:

Heart and blood vessel disease is our nation's No. 1 killer.

About half of the deaths from heart and blood vessel disease are from coronary heart disease, which includes heart attack. About 325,000 people a year die of coronary attack before they get to a hospital or in the emergency 
room. But many of those deaths can be prevented by acting fast!

Some heart attacks are sudden and intense. But most start slowly, with mild pain or discomfort.

Here are some of the signs that can mean a heart attack is happening:

Chest discomfort. Most heart attacks involve discomfort the center of the chest that lasts more than a few minutes, or that goes away and comes back. It can feel like uncomfortable pressure, squeezing, fullness or pain Discomfort in other areas of the upper body. Symptoms can include pain or discomfort in one or both arms, the back, neck, jaw or stomach.

Shortness of breath. This may occur with or without chest discomfort. Other signs. These may include breaking out in a cold sweat, nausea or light headedness.

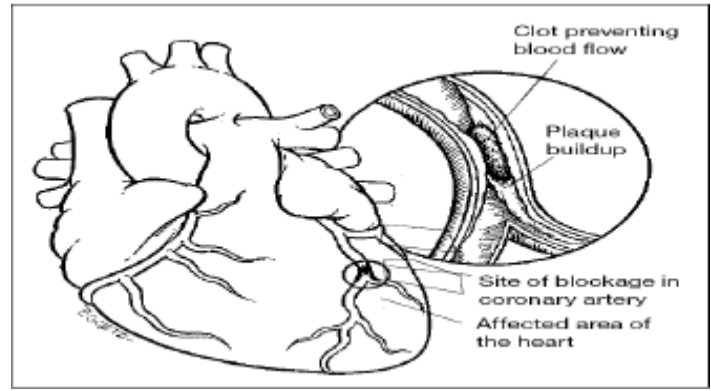

Fig.1. What causes heart attack
The senior citizens are more prone to have heart attack than young people. The Walking Stick with Heart Attack Detection is specially designed to help the senior citizens who need walking aids by walking sticks and have the most possibility of heart attack. The walking stick is used as detection unit and as the medium asking for medical help.

With combination of all three parts we can able to detect the worst condition of person at that situation gsm module call the emergency help and gps device can give the exact location of the person. In such a way person's life can be saved.

- Provide an equipment which is handy and comfortable.

- Cost effective

- Faster

\section{SYSTEM BLOCK DIAGRAM}

\section{A. Biosensors}

$\mathrm{Ag}-\mathrm{AgCl}$ ECG electrodes, we decided to use the same electrodes as our biosensors. The benefits of the electrodes include good electrical contact with human skin, low motion artifacts and strong adhesive quality to skin.

\section{B. Analog ECG Circuitry}

Based on the "Wireless Heart Attack Detector with GPS" we designed the three components, unity-gain buffers, differential amplifier and band-pass filter.
The unity-gain buffers are needed for both wrists as impedance transformers. Although skin impedance is high, the input impedance of op-amps is infinity and the opamps will be able to catch the bio signals out of the two electrodes. Differential amplifier will take the two bio signals and differentiate them with gain to get the desired ECG waveform. Band-pass filter will make sure that noise of frequencies outside $0.5 \mathrm{~Hz}$ and $150 \mathrm{~Hz}$ is eliminated. The differential amplifier gain should not exceed 33 in order to prevent a $300 \mathrm{mV}$ electrode offset potential from causing the system to saturate.

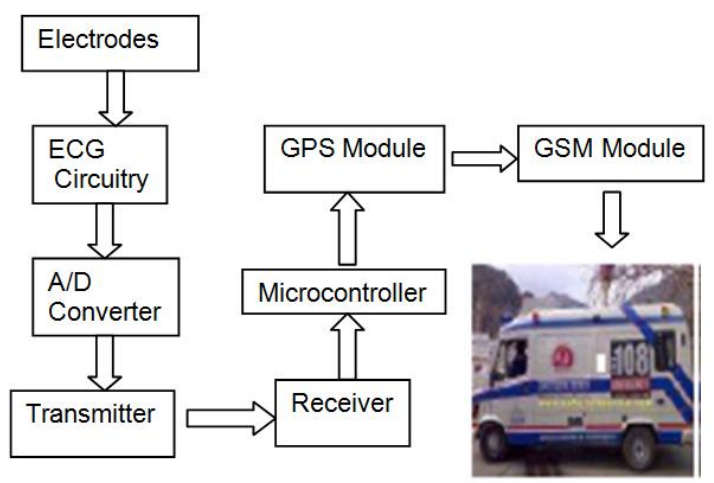

Fig.2. System Block Diagram

C. Data Transmission between Wrist and Walking Stick Our original design was to transmit the analog ECG waveform directly out of the ECG analog circuitry to the walking stick. We would use the analog signal transmission capability of the HP-3 transceiver. We wanted to make this data transmission wireless from the wrist to the stick. This would avoid the inconvenience of the stick attachment to the wrist. User can go into a car and put his stick at the back seats without detaching any wire between his wrist and the stick. He also does not have to switch the device off. When the stick falls down, it won't drag the user's wrist to the ground.

After we browsed through the data sheet of the HP-3 transceiver, we discovered that analog bandwidth of the transceiver pair is between $50 \mathrm{~Hz}$ and $28000 \mathrm{~Hz}$. As discussed in the previous project, normal ECG waveform has frequency range between $50 \mathrm{~Hz}$ and 70 Hurtz. However, to cover all the possible scenarios like sleeping and fast walking, the lower and the upper cutoff frequencies were decided to be $0.5 \mathrm{~Hz}$ and $150 \mathrm{HzSince}$ the range from $0.5 \mathrm{~Hz}$ and $50 \mathrm{~Hz}$ is outside the transmission bandwidth of the HP-3 transceiver, we thought about using mixer and oscillator to raise the lowest frequency of analog signal, which is $0.5 \mathrm{~Hz}$, to 60 $\mathrm{Hz}$. This would ensure correct transmission of the analog signal.

When we discussed this idea with our TA, we were introduced to the RS232 capability of PIC. Since RS232 signal is digital, we can use the same transceiver to transmit digital signal.

We no longer have to worry about the lowest frequency of the analog signal along with the mixer and the oscillator. 
D. A/D Conversion Of Analog ECG Signal To Digital ECG Signal

Since we are using a ARM for its RS232 feature, we thought it would be natural to use the A/D conversion feature of the ARM. We then had to decide between using an 8-bit conversion or a 10-bit conversion. 10-bit conversion would give higher resolution to the digitized ECG waveform. But, the final decision was determined by the RS232 transmission. According to the ARM Compiler Manual, when defining \#use RS232, bits sent can only be between 5 and 9 . Because 10 are not in this range, we decided to do 8-bit A/D conversion.

\section{E. Heart Attack Detection}

When the microcontroller on the walking stick has received digital ECG data from the wrists, it will check for heart attack symptoms. We decided to use the heart attack algorithm developed by the previous project. We would like to indicate to the user his heart condition so that he can take proper action like slowing down or taking a rest before heart attack really happens to him

\section{$F$. Emergency Calling}

Previous Module needed to use a Bluetooth module and a laptop to make an emergency call. Our goal was to eliminate the laptop. Our project will execute emergency calling with just a GSM based. It is wireless. The user does not have to hang his cell phone to the walking stick, making his walking stick heavy and hindering his movement.

\section{SCHEMATIC DESIGN}

\section{A. Arm Microcontroller}

The LPC2148 microcontrollers are based on a 16-bit/32bit ARM7TDMI-S CPU with real-time emulation and embedded trace support, that combine the microcontroller with embedded high-speed flash memory ranging from 32 $\mathrm{kB}$ to $512 \mathrm{kB}$. A 128 -bit wide memory interface and a unique accelerator architecture enable 32-bit code execution at the maximum clock rate. For critical code size applications, the alternative 16-bit Thumb mode reduces code by more than $30 \%$ with minimal performance penalty.

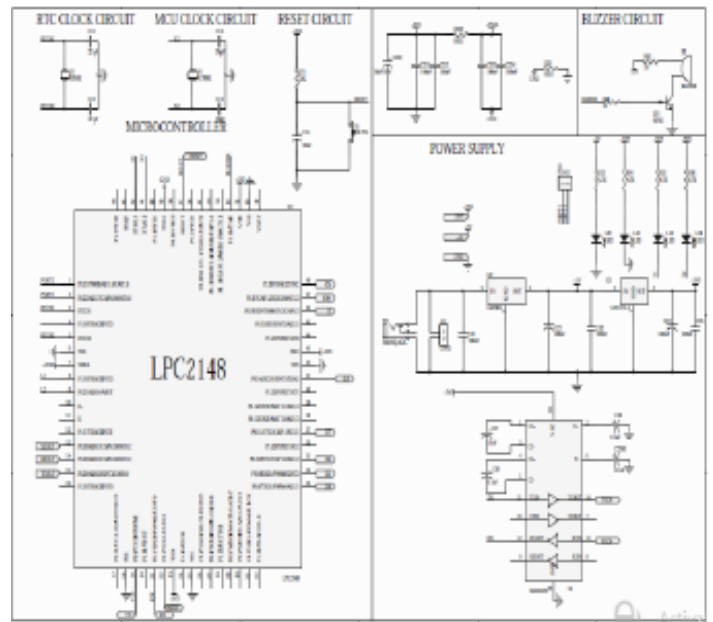

Fig.3: LPC2148 microcontroller
Due to their tiny size and low power consumption, LPC2148 are ideal for applications where miniaturization is a key requirement, such as access control and point-ofsale. Serial communications interfaces ranging from a USB 2.0 Full-speed device, multiple UARTs, SPI, SSP to I2C-bus and on-chip SRAM of $8 \mathrm{kB}$ up to $40 \mathrm{kB}$, make these devices very well suited for communication gateways and protocol converters, soft modems, voice recognition and low end imaging, providing both large buffer size and high processing power. Various 32-bit timers, single or dual 10-bit ADC(s), 10-bit DAC, PWM channels and 45 fast GPIO lines with up to nine edge or level sensitive external interrupt pins make these microcontrollers suitable for industrial control and medical systems.

\section{B. GSM Module}

A GSM modem is a specialized type of modem which accepts a SIM card, and operates over a subscription to a mobile operator, just like a mobile phone.GSM (Global system for mobile) uses a process called circuit switching. This method of communication allows a path to be established between two devices. Once the two devices are connected, a constant stream of digital data is relayed.GSM networks consist of thee major systems the Switching System (SS), The Base Station(BSS) and the Mobile station(MS).

\section{THE SWITCHING SYSTEM}

The Switching system is very operative system in which many crucial operations are conducted, SS systems holds five databases with in it which performs different functions. If we talk about major tasks of SS system it performs call processing and subscriber related functions. These databases from SS systems are HLR, MSC, VLR, AUC and EIR. The MSC in cooperation with Home Location register (HLR) and Visitor location register (VLR), take care of mobile calls and routing of phone calls. Authentication centre (AUC) is small unit which handles the security end of the system and Equipment identity register (EIR) is another important database which holds crucial information regarding mobile equipments.

\section{THE BASE STATION SYSTEM (BSS)}

The base station system have very important role in mobile communication. BSS are basically outdoor units which consist of iron rods and are usually of high length. BSS are responsible for connecting subscribers (MS) to mobile networks. All the communication is made in Radio transmission. The Base station System is further divided in two systems. These two systems, they are BTS and BSC. BTS (Base Transceiver station) handles communication using radio transmission with mobile station and BSC (Base station controller) creates physical link between subscriber (MS) and BTS, then manage and controls functions of it.

\section{MOBILE STATION (SUBSCRIBER)}

MS consist of a mobile unit and a smart card which is also referred as a subscriber Identity Module (SIM) card. This card fitted with the GSM Modem and gives the user more 
personal mobility. The equipment itself is identified by a A/D converted waveform performed as expected. The unique number known as the International Mobile most significant improvement was the emergency calling Equipment Identity (IMEI).

\begin{tabular}{|c|c|c|}
\hline \multirow{4}{*}{$\begin{array}{c}\text { GSM } \\
\text { MODEM }\end{array}$} & $\begin{array}{c}\text { Frequency } \\
\text { Band }\end{array}$ & $\begin{array}{c}\text { Quad Band } \\
850 / 900 / 1800 / 1900\end{array}$ \\
\cline { 2 - 3 } & $\begin{array}{c}\text { Transmission } \\
\text { Power }\end{array}$ & $\begin{array}{c}2 \mathrm{~W} @ 850 / 900 \mathrm{MHz} \\
1 \mathrm{~W} @ 800 / 1900 \mathrm{MHz}\end{array}$ \\
\cline { 2 - 3 } & Baud Rate & 9600 \\
\cline { 2 - 3 } & Power Supply & $12 \mathrm{~V} 1 \mathrm{~A}$ \\
\cline { 2 - 3 } & $\begin{array}{c}\text { Operating } \\
\text { Temperature }\end{array}$ & $-40^{\circ} \mathrm{C}$ to $85^{\circ} \mathrm{C}$ \\
\hline
\end{tabular}

Table 1: GSM Module Specifications

\section{A. Gps Module}

The Global Positioning System (GPS) is the only fully functional Global Navigation System (GNSS). The GPS uses a constellation of between 24 and 32 Medium Earth Orbit satellites that transmit precise microwave signals that enable GPS receivers to determine their location, speed, direction, and time. A GPS receiver receives the signals from at least three satellites to calculate distance and uses a triangulation technique to compute its two dimension (latitude and longitude) position or at least four satellites to compute its three dimension (latitude, longitude and altitude) position.

Therefore GPS is a key technology for giving device its position. GPS was developed by the United States Department of Defence. Its official name is NAVSTARGPS. It is originally used in military services but later allowed the system available free for civilian use as a common good. Since then, GPS has become a widely used aid to navigation worldwide, and a useful tool for mapmaking, land surveying, commerce, and scientific uses In This device we use a GPS receiver of HOLUX GR-67 series.

\begin{tabular}{|c|c|c|}
\hline \multirow{9}{*}{$\begin{array}{l}\text { GPS } \\
\text { Module }\end{array}$} & Chip Set & SiRF Star III Chip Set \\
\hline & $\begin{array}{l}\text { Receiver } \\
\text { Type }\end{array}$ & 20 Channels 'All in view' \\
\hline & Sensitivity & $\begin{array}{l}200,000+\text { effective correlators for } \\
\text { fast TTFF and high sensitivity } \\
\text { aquisitions }\end{array}$ \\
\hline & $\begin{array}{l}\text { Protocol } \\
\text { Format }\end{array}$ & NMEA-0183 \\
\hline & $\begin{array}{l}\text { Start up } \\
\text { times }\end{array}$ & $\begin{array}{l}\text { Hot start:1S Warm start:38S cold } \\
\text { start: } 42 \mathrm{~S}\end{array}$ \\
\hline & $\begin{array}{l}\text { Accuracy of } \\
\text { Position }\end{array}$ & 10meters 2D RMS \\
\hline & $\begin{array}{l}\text { Power } \\
\text { Requirement }\end{array}$ & 3.3 to $5.5 \mathrm{VDC}$ \\
\hline & $\begin{array}{l}\text { Working } \\
\text { Temperature }\end{array}$ & $-10^{\circ} \mathrm{C}$ to $+60^{\circ} \mathrm{C}$ \\
\hline & $\begin{array}{l}\text { Command } \\
\text { Statements }\end{array}$ & GPGGA,GPGSA,GPRMC,GPRSV \\
\hline
\end{tabular}

Table 2: GPS Parameters

\section{CONCLUSION}

The Walking Stick with Heart Attack Detection functions as designed overall. ECG waves properly collected from analog circuitry unit. The transmitting and receiving of part. We successfully deleted the laptop between Bluetooth module and the mobile phone when activating emergency calling. The wireless heart attack detector captures abnormal heart beat signals. . The alert system on the walking stick warns the user to realize his health condition. Wireless emergency calling system calls for help at the moment of heart attack via mobile phone. Electrocardiogram (ECG) signal transmitted wirelessly from the wrist to the main unit on the stick. This avoids the inconvenience of the attachment of the stick to the wrists. Automatic wireless emergency calling system.

The receiver on the stick receives the digital ECG signal, and the microcontroller runs a heart attack algorithm to detect possible heart attack symptoms. If any symptom of heart attack is detected, the risk level rises. When the risk level reaches up to the emergency mode, through GSM module, function can locate the user.

Possible future improvements are better packaging of the wrist circuitry, lower power consumption for main units. More common media rather than just walking sticks, shorter delay between heart attack detection and emergency calling via cell phone and more accurate and fast algorithm.

\section{ACKNOWLEDGMENT}

I wish to express my sincere gratitude to Prof. S. S. Gundal H.O.D. of Master of Engineering (Electronics) Amrut Vahini College of Engineering, Sangamner for providing me an opportunity to do my paper work on "Wireless Walking Stick with Heart Attack Detection ".I sincerely thank to my internal guide Prof. S. S. Kataria, for their valuable help and guidance in carrying out this paper work.

\section{REFERENCES}

[1] Hwang, Limsui, Zhao, "Wireless Heart Attack Detector with GPS", ECE445. University of Illinois ,Fall 2012 https://courses.ece.uiuc.edu/ece445/cgibin/view_project.pl?fall2004_24

[2] National Heart, Lung, and Blood Institute, Disease and Condition Index, "What Is a Heart Attack?", August2014. http://www.nhlbi.nih.gov/health/dci/Diseases/HeartAttack/HeartAtt ack_WhatIs.html

[3] National Heart, Lung, and Blood Institute, Disease and Condition Index, "What Is Coronary Artery Disease?",August2011, http://www.nhlbi.nih.gov/health/dci/Diseases/Cad/CAD_WhatIs.ht $\mathrm{ml}$

[4] ECG Library. http://www.ecglibrary.com/ecghome.html

[5] Wikipedia, the Free Encyclopedia, "RS-232", http://en.wikipedia.org/wiki/RS-232

\section{BIOGRAPHY}

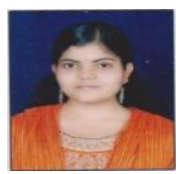

Miss. Harsha C.Puranik is currently Working as a Lect. At Electronics and tele-communication dept. of IOK COE, Pune.She has done her B.E. in Electronics \& comm..from S.S.V.P.S. COE in 2010 and M.E.(II ${ }^{\text {nd }}$ year Appearing ) from Vahini college Engineering sangamner. 